\title{
Advances towards reliable identification and concentration determination of rare cells in peripheral blood
}

R. Alemany Server, D. Martens, K. Jans, P. Bienstman, D. Hill

R. Alemany Server, D. Martens, K. Jans, P. Bienstman, D. Hill, "Advances towards reliable identification and concentration determination of rare cells in peripheral blood," Proc. SPIE 9705, Microfluidics, BioMEMS, and Medical Microsystems XIV, 97050G (18 March 2016); doi: 10.1117/12.2218621

SPIE. Event: SPIE BiOS, 2016, San Francisco, California, United States 


\title{
Advances towards reliable identification and concentration determination of rare cells in peripheral blood
}

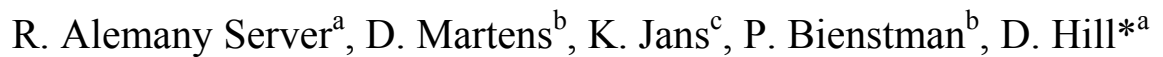 \\ ${ }^{a}$ Instituto de Ciencias de los Materiales, Universidad de Valencia, PO Box 22085, 46071 Valencia, \\ Spain; ${ }^{b}$ Center for Nano- and Biophotonics (NB-Photonics), Ghent University-IMEC, Sint- \\ Pietersnieuwstraat 41, 9000 Gent Belgium; ${ }^{\mathrm{C}}$ IMEC vzw, Kapeldreef 75, 3001 Leuven Belgium
}

\begin{abstract}
Through further development, integration and validation of micro-nano-bio and biophotonics systems FP7 CanDo is developing an instrument that will permit highly reproducible and reliable identification and concentration determination of rare cells in peripheral blood for two key societal challenges, early and low cost anti-cancer drug efficacy determination and cancer diagnosis/monitoring.

A cellular link between the primary malignant tumour and the peripheral metastases, responsible for $90 \%$ of cancerrelated deaths, has been established in the form of circulating tumour cells (CTCs) in peripheral blood. Furthermore, the relatively short survival time of CTCs in peripheral blood means that their detection is indicative of tumour progression thereby providing in addition to a prognostic value an evaluation of therapeutic efficacy and early recognition of tumour progression in theranostics. In cancer patients however blood concentrations are very low (=1 CTC/1E9 cells) and current detection strategies are too insensitive, limiting use to prognosis of only those with advanced metastatic cancer. Similarly, problems occur in therapeutics with anti-cancer drug development leading to lengthy and costly trials often preventing access to market.
\end{abstract}

The novel cell separation/Raman analysis technologies plus nucleic acid based molecular characterization of the CanDo platform will provide an accurate CTC count with high throughput and high yield meeting both key societal challenges. Being beyond the state of art it will lead to substantial share gains not just in the high end markets of drug discovery and cancer diagnostics but due to modular technologies also in others. Here we present preliminary DNA hybridization sensing results.

Keywords: Circulating Tumour Cells, silicon nitride waveguides, Mach Zhender Interferometry, Multimode Interference, Arrayed Waveguide gratings, AlOx sacrificial layer, azide organosilanes, DNA hybridization,

\section{CTC DETECTION TECHNOLOGIES}

\subsection{A Point of Care Diagnostic Challenge}

Some diagnostic applications are extremely demanding, such as those requiring both the identification and concentration determination of extremely low levels of cells in peripheral blood, that to date no such point of care (PoC) diagnostic technology generic or otherwise exists for these. One such application is for the recognition and the concentration measurement of rare circulating tumour cells (CTCs) for early diagnosis staging and monitoring of cancer. Pancreatic cancer is one such target, and is of strong socio-economic interest as currently it is usually diagnosed at an advanced stage when rapid progression has occurred for widespread disease dissemination and so a $96 \%$ chance of death for some 68000 Europeans diagnosed every year. A powerful tool for correct diagnosis and staging of pancreatic cancer would permit not just advances in cancer management, where clinicians could prescribe a suitable early treatment and monitor its progress within theranostics, but also in drug discovery for quick and accurate determination of the effectiveness of new anti-cancer drugs in development tests.

\subsection{State of art}

The detection and molecular characterization of CTCs are one of the most active areas of translational cancer research, with $>400$ clinical studies having included CTCs as a biomarker [1]. There is a battery of analytical tools available for

Microfluidics, BioMEMS, and Medical Microsystems XIV, edited by Bonnie L. Gray, Holger Becker Proc. of SPIE Vol. 9705, 97050G · @ 2016 SPIE · CCC code: 1605-7422/16/\$18 · doi: 10.1117/12.2218621 
the analysis and characterization of cells once isolated, including immunochemistry and nucleic acid analysis. However, CTCs are rare even in patients with advanced cancer, as low as 1 CTC in 1 billion blood cells. Furthermore, the techniques currently used to isolate these CTCs require laborious manual sample preparation steps that result in highly variable results and low sensitivity. A reliable rare cell sorter technology for CTCs is therefore exceedingly challenging. Some of the current commercially available CTC isolation methods are described in Table 1.

Table 1: CTC detection technologies

\begin{tabular}{|c|c|c|c|c|}
\hline \multirow[t]{2}{*}{ Technology Name } & \multirow[t]{2}{*}{ Company Name } & \multirow[t]{2}{*}{ Isolation Method/ Assay } & \multicolumn{2}{|c|}{ Detection Method } \\
\hline & & & $\begin{array}{c}\text { CTC } \\
\text { Count }\end{array}$ & $\begin{array}{c}\text { Molecular } \\
\text { Analysis }\end{array}$ \\
\hline AdnaTest & AdnaGen, & Immuno/Enrichment + Characterization & No & Yes \\
\hline ApoStreamTM & ApoCell, Inc & Electrical + Immuno/Enrichment & Yes & No \\
\hline IsoFlux & $\begin{array}{l}\text { Fluxion Bioscience, } \\
\text { Inc }\end{array}$ & $\begin{array}{l}\text { Electrical + Immuno/Enrichment + } \\
\text { Characterization }\end{array}$ & Yes & No \\
\hline CellSearchTM & Veridex & Immuno/ Enrichment + Enumeration & Yes & No \\
\hline CaptorTM & Abnova, Taipei, & $\begin{array}{l}\text { Size/Enrichment }+ \text { Enumeration }+ \\
\text { Characterization }\end{array}$ & Yes & No \\
\hline DEPArray & Silicon Biosystems, & Electrical/ Enumeration & Yes & No \\
\hline Epic HD-CTC Assay & Epic Science, Inc & Immuno/Enumeration + Characterization & Yes & No \\
\hline CellCollector & GILUPI & $\begin{array}{l}\text { Invivo (immuno)/Enrichment }+ \text { enumeration } \\
\text { Enumeration }+ \text { Characterization }\end{array}$ & Yes & No \\
\hline OncoCEE-BR TM & Biocept, Inc & Immuno/Enumeration + characterization & Yes & No \\
\hline Cytotrack CT4 & CytoTrack ApS & Immuno/Characterization & Yes & No \\
\hline ScreenCell & Screen Cell, & Size/Enrichment + characterization & Yes & No \\
\hline CanDo platform & CanDo Consortium & $\begin{array}{l}\text { size }+ \text { Immuno/Enrichment }+ \text { enumeration }+ \\
\text { characterization }\end{array}$ & Yes & Yes \\
\hline
\end{tabular}

The current "gold standard" for CTC isolation and CTC enumeration is the Cellsearch ${ }^{\circledR}$ System by Veridex, an affiliate of Johnson \& Johnson. The CellSearch ${ }^{\circledR}$ System is the first and only $510(\mathrm{k})$ diagnostic test used to automate the capture and detection of CTCs, tumour cells that have detached from solid tumours and entered the patient's blood. The CellSearch System is focused on isolating CTCs from the peripheral blood using immunomagnetic beads conjugated with EpCAM [2]. This FDA approved technology sets the standard for patients with metastatic breast, prostate or colorectal cancer. Patients in the non-metastatic setting or with other tumour indications are not eligible to benefit from this technology. However, its success has been seriously hampered by very low yield and purity of isolated CTCs [3]. The low yield of immunomagnetic isolation of CTCs is likely to reflect the fact that these cells are very rare and do not readily survive the multi-step batch purification technique involved. A reliable clinical test that can confirm that a patient is free of circulating tumour cells is of great value for the patient in a staging situation or in a situation of follow up after successful treatment.

All-in-all current commercial technologies are semi-automated at best with multiple "bulk" enrichment steps such as magnetic particle sorting. As a result, the sensitivity and specificity of CTC detection and analysis is very low, highly variable, and not very useful for a clinical test.

\subsection{Moving beyond state of art}

In CanDo, through the integration of various components and sub-systems recently developed in other projects a diagnostic platform is targeted that will enable accurate and reliable early pancreatic cancer diagnosis, monitoring and prognosis determination based on efficient capture and recognition of CTCs in peripheral blood at unprecedented limits of detection.

The targeted approach (Figure 1) is based on a disposable cartridge system that combines a powerful cell separation technology; principally through a combination of the GILUPI system [4] or inertial microfluidics [5] and high yield sensitive immuno-capture, with Raman spectroscopy to identify/quantify the CTC and sensitive nanophotonic sensors for nucleic acid analysis from the lysed CTCs for molecular characterization. Not only should the sensors be sensitive but 
they and their instrumentation should also be capable of multiplexing of up to 20 times and be of a suitably low cost to make the overall platform competitive. In this paper we present some preliminary results of some novel sensors that would meet such criteria.

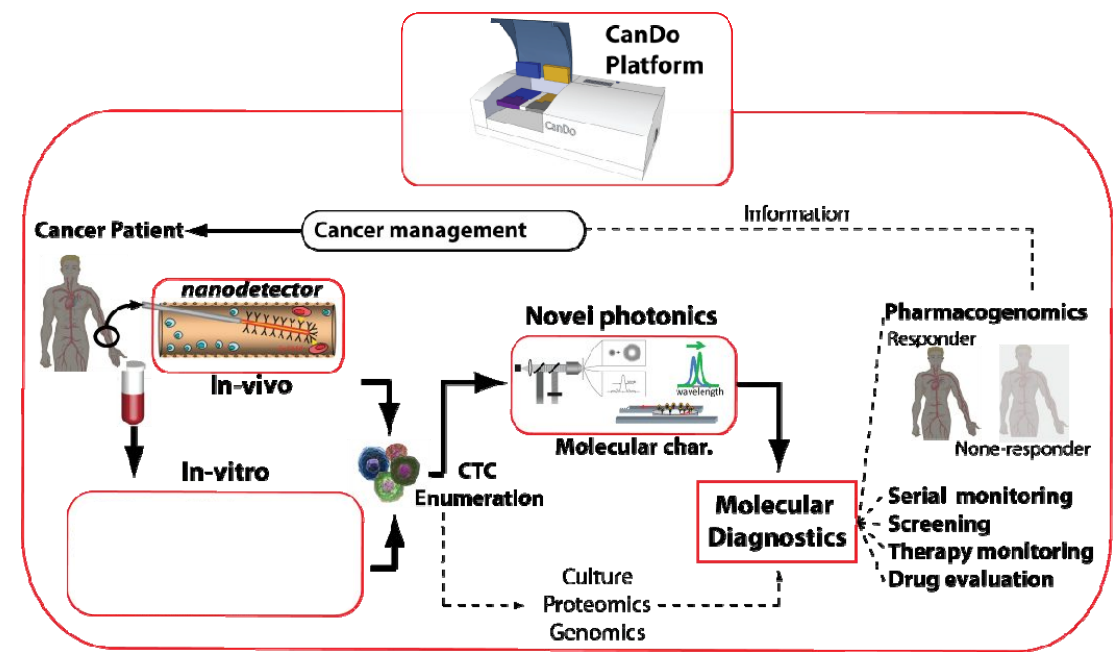

Figure 1. A schematic of the principally targeted CanDo platform with indicated components and sub-systems. The platform achieves cancer diagnosis through CTC capture and enumeration (cellular characterization) followed by nucleic acid analysis (molecular characterization). It takes whole blood from cancer patient and isolates the CTCs using a novel in-vivo isolation technology (whilst another in-vitro technology is considered as a backup) to deliver cell enumeration. The enumerated cells are then further processed for nucleic acid characterization using a label-free photonic sensor array.

\section{NANOPHOTONIC BIOSENSORS}

\subsection{State of art}

Biosensors are by definition a combination of a biological receptor molecule and a physical or physicochemical transducer [6]. The main challenge for biosensor technologies in diagnostics and other biosensing applications is the rapid detection of low concentration of a specific analyte in small sample volumes typically in a complex mixture with large background signals (a problem known as non-specificity).

Optical methods of transduction offer important advantages such as non-invasive/safe, label free and multi-dimensional (intensity, wavelength, phase, polarization) high sensitivity detection across optical frequencies that coincide with a wide range of physical properties of bio-related materials. Within the important category of label free biosensing, optical transducers have a significant presence: indeed, the dominant vendor of label free technology is Biacore, and their technology is a photonic one, based on surface plasmon resonance. However, the detection limit of this technology is inherently limited due to absorption effects in the metal. Nevertheless, with measurements of optical absorption and refractive index (RI) being well established analysis methods in chemistry and biology they have been incorporated in label-free biosensor based lab-on-chip solutions through technologies such as surface plasmon resonance (SPR) [7][8] as well as others based on thin-film interference spectroscopy [9], or optical waveguides [10].

In the quest for label free lab-on-a-chip systems with lower limits of detection an alternative optical biosensing technology to SPR and its metallic structures has arisen based on dielectric structures [11]. Specifically, recent maturity in silicon photonics technology that operates in the near infra-red (NIR) has led to advances in the employment for sensing of the evanescent field of guided modes in high-index core dielectric waveguides such as interferometers [12], photonic crystals [13], microsphere resonators [14] and silicon ring resonators - the latter having achieved detection limits down to the femtomolar range for proteins [15]. Their compatibility with mature silicon microprocessing technologies and materials have permitted a high integration of complex structures, on-chip integration of electronics and, via the addition of polymers, microfluidic channels for high-throughput arrays on a single-chip for simultaneous detection of multiple analytes. These devices with optical transmission lengths in the order of millimetres achieve 
surface concentration detection limit (SCDL) values in the order of $0.1-1 \mathrm{pg} / \mathrm{mm}^{2}$ and so in recent years, the EU has funded relevant projects regarding optical biosensing systems based on these [16]. Table 2 summarises the results from two of these, SABIO [17] and InTopSens [18].

Table 2: A summary of main achievements and problems encountered in two recent EC projects. UPV corresponds to the group of A. Maquieira at the Technical University of Valencia and IMEC to that of T. Stakenborg at IMEC.

\begin{tabular}{|c|c|c|c|}
\hline Project & Technology & Main Achievements & Problems encountered \\
\hline SABIO & $\begin{array}{c}\text { SiN slot ring } \\
\text { resonators with } \\
\text { UPV chemistry }\end{array}$ & $\begin{array}{c}\text { Methanol/Ethanol dilution series detection: } 5 \times 10^{-6} \mathrm{RIU} . \\
\text { Glutaraldehyde-AntiBSA detection: successful detection of } \\
0.9 \mathrm{pg} / \mathrm{mm}^{2} \text {, a significant improvement on a previously } \\
\text { published value of } 28 \mathrm{pg} / \mathrm{mm}^{2} \text {. }\end{array}$ & $\begin{array}{c}\text { Poor resolution due to } \\
\text { fabrication technology } \\
\text { limitations (sidewall } \\
\text { roughness). }\end{array}$ \\
\hline InTopSens & $\begin{array}{c}\text { Si slot ring } \\
\text { resonators with } \\
\text { UPV chemistry }\end{array}$ & $\begin{array}{c}\text { Aqueous salt solution detection: } 4.2 \times 10^{-5} \mathrm{RIU} \text { Biotin-avidin } \\
\text { detection: } 5 \mathrm{pg} / \mathrm{mm}^{2}\end{array}$ & $\begin{array}{c}\text { Lower resolution than SABIO } \\
\text { device principally due to } \\
\text { bending and mismatch losses. }\end{array}$ \\
\hline InTopSens & $\begin{array}{c}\text { Si ring resonators } \\
\text { with UPV chemistry }\end{array}$ & $\begin{array}{c}\text { Aqueous salt solution detection: } 5 \times 10^{-6} \text { RIU Biotin-avidin } \\
\text { detection: } 2 \mathrm{pg} / \mathrm{mm}^{2} \text {. High degree of multiplexing achieved. }\end{array}$ & $\begin{array}{c}\text { Instrumentation cost very } \\
\text { expensive (tunable laser). }\end{array}$ \\
\hline InTopSens & $\begin{array}{c}\text { Vernier cascade Si } \\
\text { ring resonators with } \\
\text { UPV chemistry }\end{array}$ & $\begin{array}{c}\text { Aqueous salt solution detection: } 8.3 \times 10^{-6} \text { RIU. DNA } \\
\text { detection: Cheaper readout but poorer LoD than regular ring } \\
\text { resonators. Inherently temperature stable. }\end{array}$ & $\begin{array}{c}\text { Surface chemistry issues } \\
\text { prevented successful detection } \\
\text { of on-chip hybridisation. }\end{array}$ \\
\hline Post & $\begin{array}{c}\text { Regular Si ring } \\
\text { resonators with } \\
\text { IMTC chemistry }\end{array}$ & $\begin{array}{c}\text { DNA detection: } 100 \mathrm{nM} 25 \text { bp, to be compared to the best } \\
\text { published result of } 10 \mathrm{pM} \text { of } 58 \text { mers for a biomodal } \\
\text { interferometer [19]. }\end{array}$ & $\begin{array}{c}\text { Limitation to multiplexing } \\
\text { capability. }\end{array}$ \\
\hline
\end{tabular}

However, none of these have been commercialized and it is debatable whether, the Maverick Technology, based on (non-slot waveguide) ring resonators fabricated by IMEC, of Genalyte will be a commercial success for the same reason - they require a high performance and therefore costly tuneable laser. In the case of the Genalyte system, its sale cost is anywhere between 50 and $100 \mathrm{k} €$ which automatically limits it to niche applications, almost all being non-point of care, across healthcare, food safety, environment, security markets. Low cost ring resonator technologies such as the 'Verniers' [20] with their cheap broadband light sources have similar sensitivities but their on-chip spectrum analysers with resolutions of only $1 \mathrm{~nm}$ are no match for the expensive external spectrum analysers, resulting overall in poorer limits of detection. Moreover, they are more limited for multiplexing.

\subsection{Moving beyond state of art}

\subsection{1. $\mathrm{Si}_{3} \mathrm{~N}_{4}$ integrated unbalanced Mach-Zehnder Interferometer}

Photonic biosensors (Figure 2) based on a silicon nitride $\left(\mathrm{Si}_{3} \mathrm{~N}_{4}\right)$ integrated unbalanced Mach-Zehnder Interferometer (MZI) have been developed in the FP7 POCKET project by two of us (Mertens and Bienstman). Such biosensors have been shown to have both sufficient multiplex capability and limit of detection for the CanDo application and cost up to $15 \mathrm{x}$ less than the aforementioned technologies in section 2.1 that operate at wavelengths of 1300 or $1550 \mathrm{~nm}$. In order for their implementation to be low cost, an $850 \mathrm{~nm}$ free space SLED broadband source has been used as the incident light and, an on-chip arrayed waveguide grating (AWG) [21], filters the output MZI spectrum prior to the light exiting the chip. Specifically, the light is coupled into the on chip optical circuit through a $16^{\circ}$ vertical grating coupler, before being guided along a monomode strip waveguide until its division between two arms using a MMI coupler. The whole circuit is covered with an oxide cladding layer except the sensing waveguide which is exposed to the analyte. The light from both arms are combined using a second MMI coupler, so there is an intensity modulation at its output depending on the phase shift introduced by the sample to be measured over the sensing branch. The MZI output is then filtered through the AWG into 30 channels with $1 \mathrm{~nm}$ bandwidth each, and the light is coupled out of the circuit over 30 grating couplers. The output optical intensity from the couplers is measured by a CCD camera over a lens, and proprietary software fits peaks to the spectra and represents the MZI output modulation. The different designs of the MZI biosensors (MZI1, MZI2, MZI3 etc) on each chip in the reported experiments (Figure 3) permitted different sensitivities. Each chip contained 7 Input grating couplers, 6 different sensors, 7 AWG filters, 210 output grating couplers ( 6 sensors by 30 output AWG channels and 30 more for the reference) with the input and output couplers orthogonal for reducing scattering. Although each chip of biosensors used was identical those functionalised in one batch were labelled as HULKx and those in 
another SPIDERMANy where $\mathrm{x}$ and $\mathrm{y}$ are numbers that indicate the sequence of use, first HULK1 and then HULK2 in the next experiment etc.

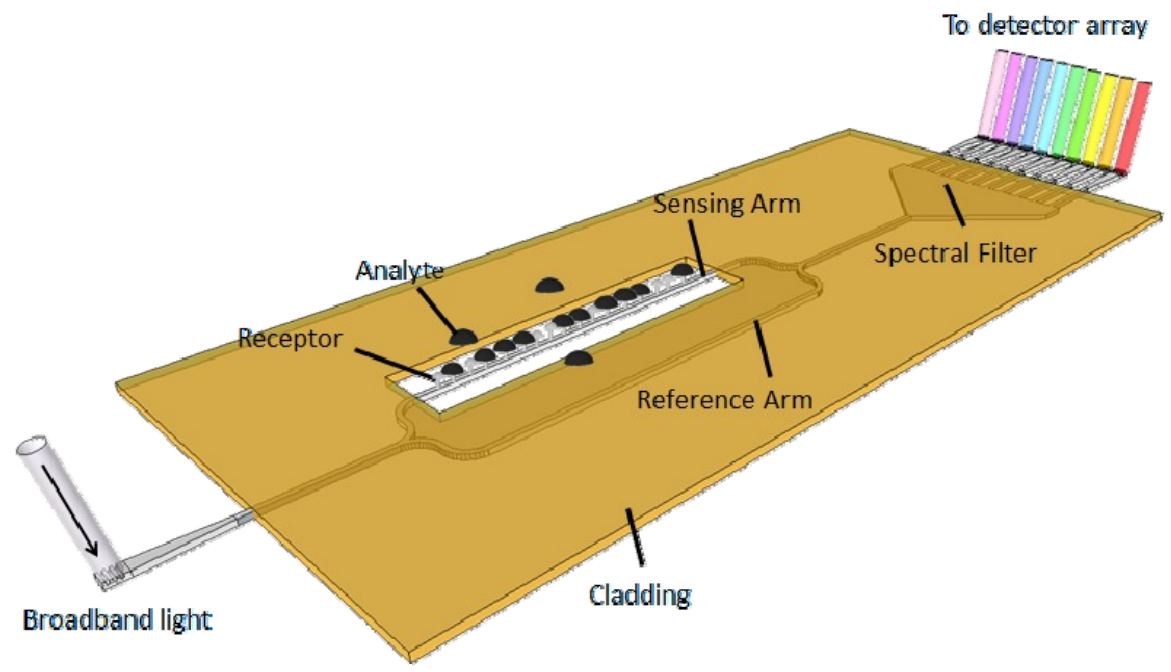

Figure 2. Schematic of the nanophotonic integrated biosensor circuit.

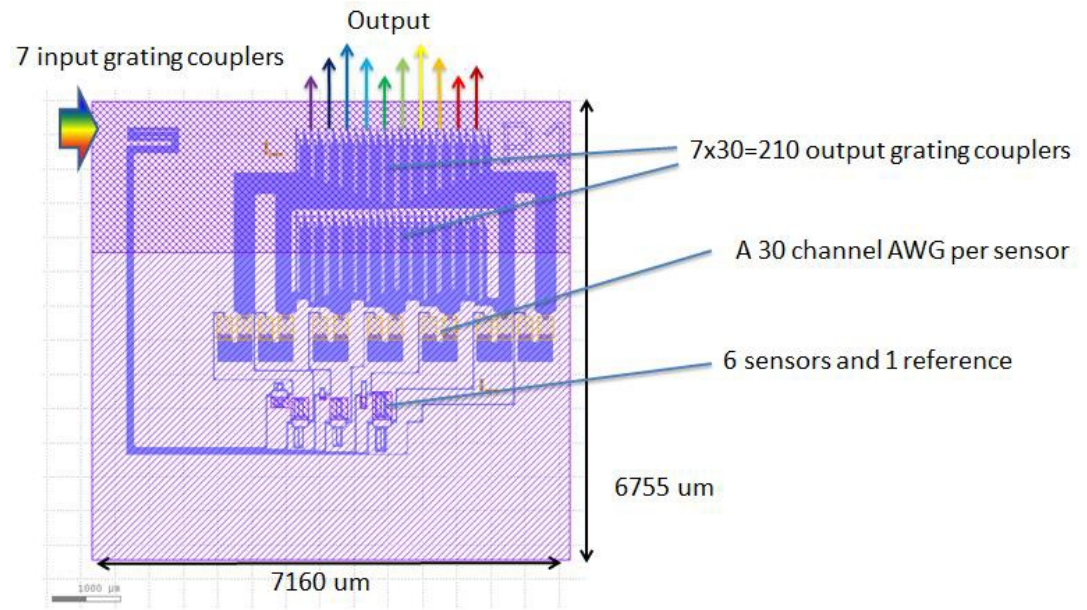

Figure 3. Components distribution of the fabricated and analysed chip

\subsubsection{DNA sensor functionalisation}

Synthetic hexynyl-modified single strand DNA (ssDNA) probes were covalently bonded to the functionalised surface of the MZI sensing waveguides through the use of standard click chemistry. The surface was functionalised with azide organosilanes previously immobilized using a vapour phase deposition method $\left(145^{\circ} \mathrm{C}, 25 \mathrm{mbar}\right)$.

\subsubsection{Experimental setup}

The setup (Figure 4) includes a syringe pump to flow a hybridization buffer $(1 \mathrm{M} \mathrm{NaCl}, 10 \mathrm{mM}$ Tris.HCl, and $2 \mathrm{mM}$ EDTA; pH 7) at $10 \mu \mathrm{L} /$ min towards the chip via a valve which is switched to allow the ssDNA analyte to flow through an inlet tube and into a PDMS (PolidimetilSiloxane) microfluidic channel on the surface of the chip that conducts the sample over the surface of the sensing arm waveguide. The output of the microfluidic channel thereafter passes into an outlet tube and onto the waste chamber. The chip is mounted on an aluminium plate with a Peltier element which is connected to a temperature controller to fix the chip temperature to $303.45 \mathrm{~K}$. The optical source with a collimator is 
mounted on a three axis micro-positioner to align the light spot onto the input grating couplers of the chip. The intensity of the 30 output light spots is measured by a CCD camera and the control computer software detects the wavelength shifts.

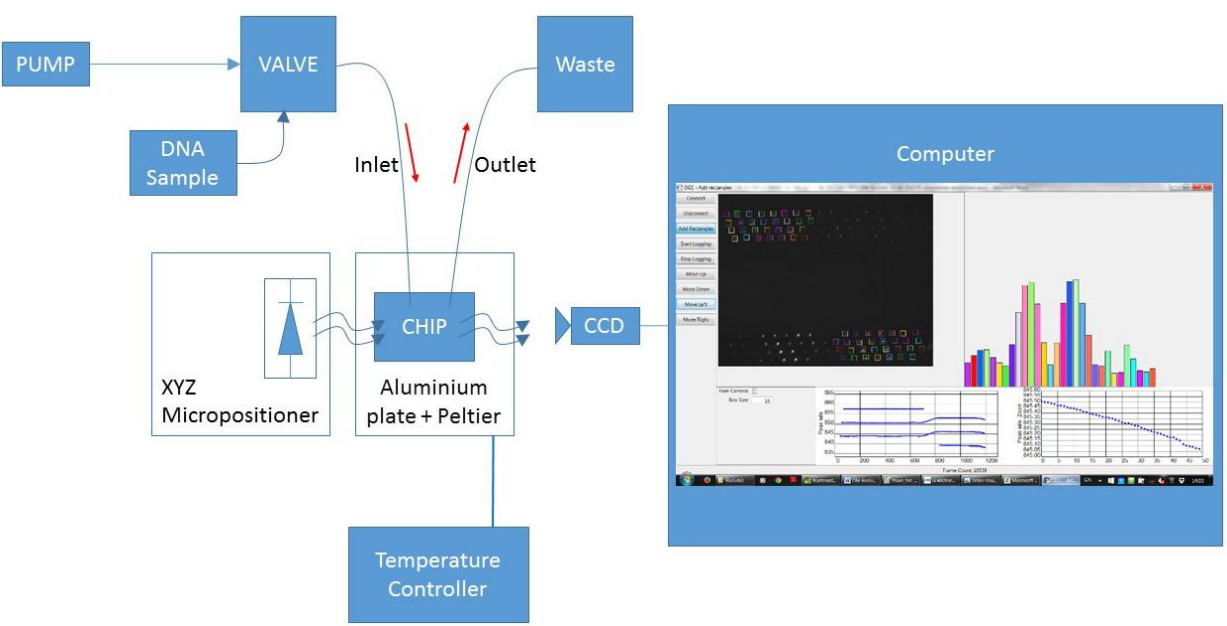

Figure 4. Schematic of the DNA detection setup

\subsubsection{Experimental measurements}

Before the start of DNA detection, the sensing surfaces within the microfluidic system were rinsed with injections of isopropanol and distilled water followed by $\mathrm{HCl}$ to regenerate the surface. Afterwards, the DNA hybridization buffer was injected at a rate of $10 \mu \mathrm{L} / \mathrm{min}$ to allow the surface of the waveguide to capture the complementary ssDNA analyte. Once the peak wavelength was stable, and with the buffer still flowing, injections of the analyte (a 25 mers complementary ssDNA) at concentrations of $5 \mathrm{pM}, 50 \mathrm{pM}, 500 \mathrm{pM}, 5 \mathrm{nM}, 50 \mathrm{nM}, 400 \mathrm{nM}$ were introduced, each separated by $\mathrm{HCl}$ injections to regenerate the surfaces.

Figure 5 shows sensorgrams for four different DNA concentrations on four different sensors within chips Spiderman1, 2 and 3. Figure 5a shows the detection of just 5pM of ssDNA from a wavelength shift of 50pm although the noise level is around $18 \mathrm{pm}$.

Figure 6a shows the measured peak wavelength shift over different DNA concentrations, from 50pM upwards, on MZI4 and MZI6 sensors (both on chip HULK4). From linear fits to this preliminary data a 23pm/pM sensitivity was derived for the sensor MZI4, and 37pm/pM for MZI6. Figure $6 \mathrm{~b}$ shows measurements from an experiment with a sensor of the same design (MZI4) on a chip functionalised in a separate batch, Spiderman4. In this experiment $\mathrm{HCl}$ was not injected between samples, and so the DNA concentration range extends up to $455 \mathrm{nM}$. From a linear fit over the first three data, the derived sensitivity is similar to that of that on Hulk4 at $17 \mathrm{~nm}$, with the difference possibly due to differences in surface activity being functionalised in two different batches.

The wavelength dependency on temperature has also been studied. Figure 7 shows that MZI4 on the Spiderman 4 chip has a temperature sensitivity of $-54.9 \mathrm{pm} / \mathrm{K}$. 


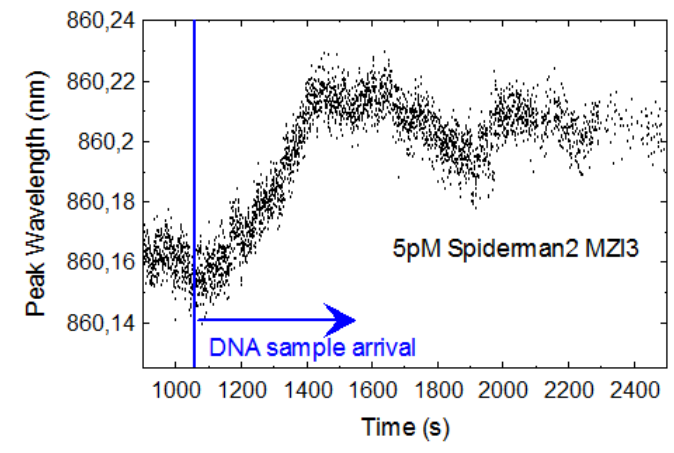

a

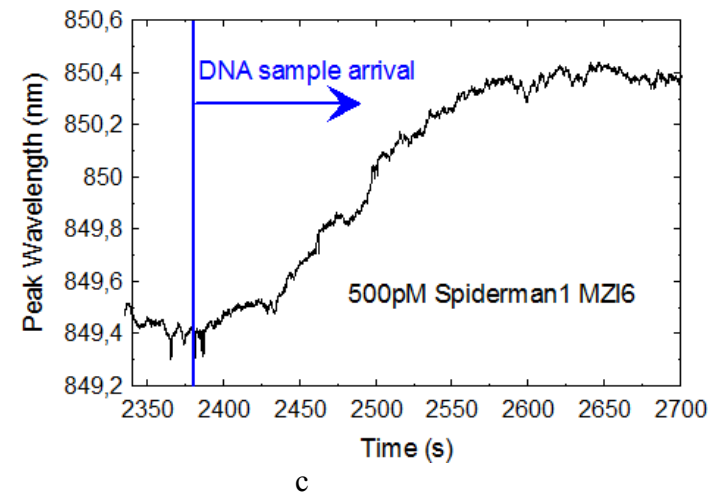

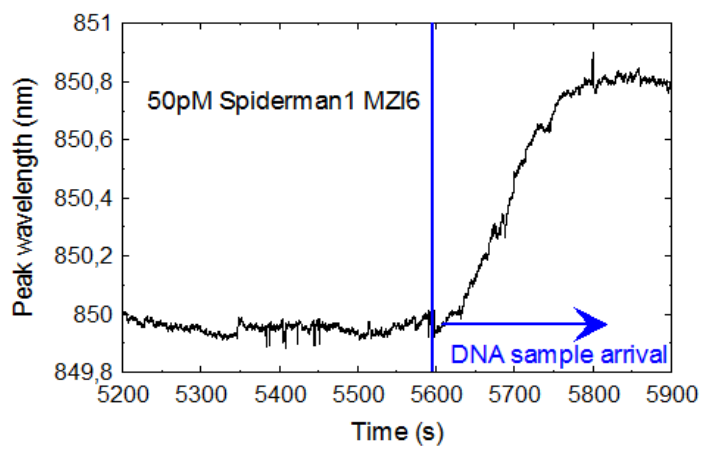

b

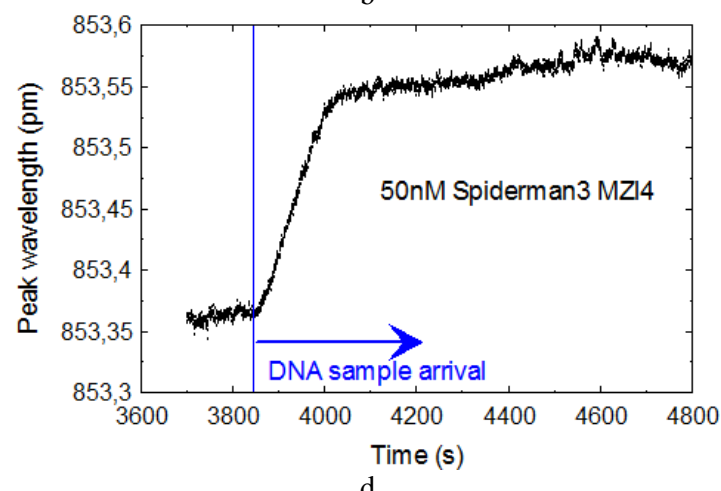

d

Figure 5. Measured sensorgrams at different concentrations of 25-mers DNA fragments on different sensors.
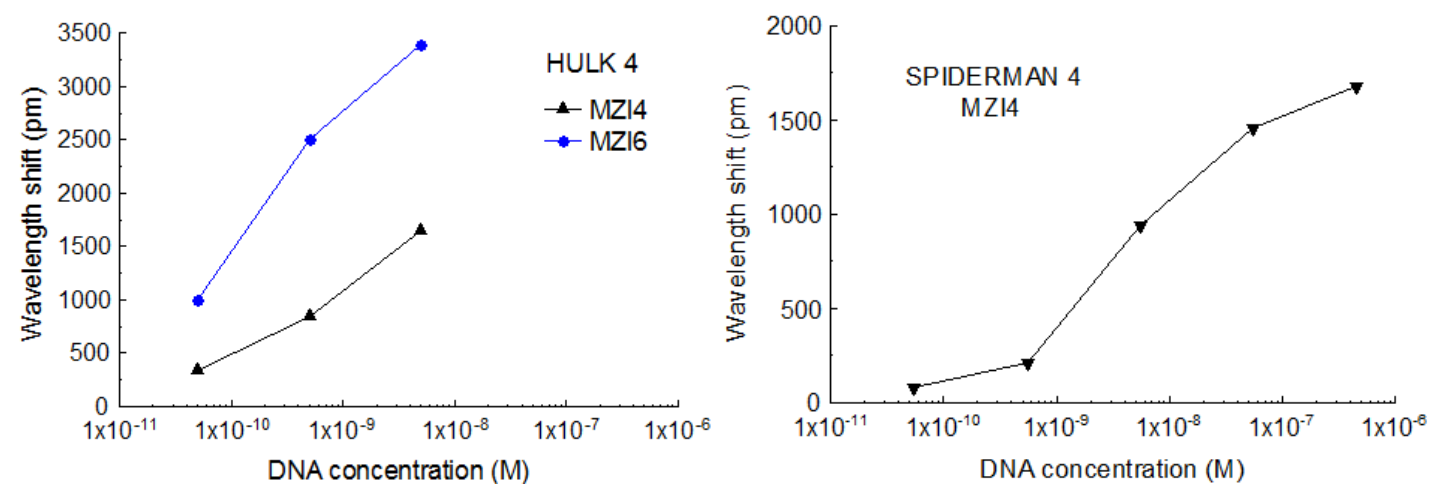

Figure 6. Measured wavelength shifts for given ssDNA analyte concentrations for sensors MZI4 and MZI6 on chip Hulk4 with HCl injections between samples (a), and MZI4 on chip Spiderman4 without $\mathrm{HCl}$ injections between samples (b).

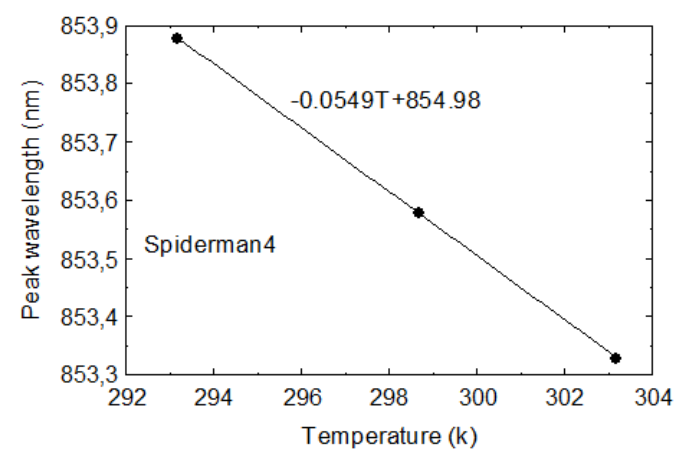

Figure 7. Peak wavelength over temperature for MZI4 on chip Spiderman4. 


\section{CONCLUSIONS}

A low cost, potentially highly multipliexed biosensor design based on an integrated unbalanced silicon nitride nanophotonic Mach-Zehnder Interferometer integrated together with a spectrum analyser is presented, and its functionalization for DNA detection described. As far as we are aware this is the first time experimental results for DNA hybridization sensing have been shown for such a device. If the preliminary result of 5pM detection of 25mers ssDNA is confirmed in further experiments underway for full analytical data, it would be superior than what we understand to be the best published limit of detection to date of $10 \mathrm{pM}$ of 58 mers. That with said the preliminary data is sufficient to demonstrate the suitability of the biosensors for their integration into the CanDo device.

\section{ACKNOWLEDGMENTS}

We thank the INTEC group from the University of Ghent for the design of the sensor and IMEC for its functionalization. This work was carried out within the FP7-ICT-610472-CanDo project, funded by the European Commission.

\section{REFERENCES}

[1] Catherine Alix- Panabières and Klaus Pantel. Clinical Chemistry (2013) 59:1 110-118

[2] Kagan, M., et al., Cytometry Part A, 2004. 59 A (1): p. 64-64.

[3] Allard, W.J., et al., Clin Cancer Res, 2004. 10(20): p. 6897-90

[4] The GILUPI detector Cancer01 is CE approved (Directive 93/42/EEC AnnexV, Reg. No. DD600794640001, date 2012-10-17) by TÜV Rheinland, Germany

[5] A. Russom, et al, New J. of Physics 11, 75025. 2009

[6] A. Brecht and G. Gauplitz, Biosensors and Biolectronics 10 (1995) 923-936

[7] H.J. Lee, A.W. Wark, R.M. Corn. Langmuir, 22 (2006) pp5241-5250.

[8] Y.A. Li, A.W. Wark, H.J. Lee, R.M. Corn. Anal. Chem. 78, (2006), pp3158-3164.

[9] J. Lu, et al.. Anal. Chem. 76, (2004) pp4416-4420.

[10] J. Voros, et al., Biomaterials 23 (2002) pp3699-3710.

[11] G. Gauglitz. Anal. Bioanal. Chem, 381 (2005), pp141-155

[12]D. Erickson et al., Journal of Microfluidics and Nanofluidics 4, 33-52 (2008)

[13]F. Vollmer, and S. Arnold, Nature Methods 5, 591-596 (2008)

[14] M. C. Estevez, M. Alvarez, and L. M. Lechuga, Laser \& Photonics Reviews, 6(4), 463487 (2012)

[15] M. Iqbal et al., IEEE Journal of Selected Topics in Quantum Electronics 16 (3), 654-661 (2010)

[16]FP5 BIOMIC - http://cordis.europa.eu/search/index.cfm?fuseaction=proj.document\&PJ RCN=5158146

[17] IST-2006-026554 (SABIO), http://www.ist-sabio.org/

[18] ICT-2007- 223932. (INTOPSENS), http://www.ee.kth.se/intopsens/

[19] K. Zinoviev, et al., Adv. Opt. Tech. 2008 (Article ID: 383927), 6 p (2008).

[20] T. Claes, W. Bogaerts, P. Bienstman, Opt Lett. 36(17), 3320-3322. (2011)

[21]D. Martens, et al., IEEE PTL, Vol. 27, No. 2, January 15 (2015) 